DOI 10.18551/rjoas.2019-11.09

\title{
CONSUMERS' CHOICE AND WILLINGNESS TO PAY FOR BIOFORTIFIED FOODS IN GHANA: A STATED PREFERENCE APPROACH
}

\author{
Owusu Rebecca \\ Department of Agricultural Economics and Extension, University of Cape Coast, Ghana \\ E-mail: rebecca.owusu@ucc.edu.gh
}

\begin{abstract}
Micronutrient malnutrition is a major problem in developing countries, especially vitamin $A$ deficiency. Alternative methods of addressing vitamin A deficiency have been introduced including Biofortification. In this paper, a discrete choice experiment method is used to examine consumer preferences for biofortified rice in Ghana. Specifically, a mixed logit and the generalized multinomial logit models are used to examine consumer preferences and willingness to pay for biofortified rice using a sample of 153 consumers. The econometric modeling revealed that on average, consumers prefer biofortified rice with vision attribute that completely removes blindness, has small grains and is relatively cheap. The results further show that preferences are different across gender groups such as the young, males and females and these variations have to be considered in breeding biofortified crops for consumers.
\end{abstract}

\section{KEY WORDS}

Biofortified rice, choice experiment, consumer preference, vitamin A deficiency, malnutrition, developing countries.

Micronutrient malnutrition, a condition resulting from insufficient vitamins and minerals in the diets of people is a widespread and serious problem, especially in developing countries (González, Johnson, \& Qaim, 2009). Research shows that micronutrient malnutrition has health implications such as mental and physical disorders, retardation and blindness (Chowdhury, Meenakshi, Tomlins, \& Owori, 2011; Ritchie \& Roser, 2017). The malnutrition or deficiency mainly relates to micronutrients such as iron, calcium, iodine and Vitamin A. For example, it has been estimated that about 250 million pre-school children suffer from vitamin A deficiency (VAD), out of which $10 \%$ go blind and half die annually (Christian et al. 2006). In Ghana, about $76 \%$ of the population suffers from VAD, which is almost twice Africa's average of 41.49 (Taylor 2014).

Over the years, intervention programs have been initiated to address problems of VAD. Such interventions included nutritional education programmes, supplementation using vitamin A capsules and drops (Domonko, McFadden, Mishili, Mullally, \& Farnsworth, 2018). However, the food supplementation programmes have not been very effective because of the high costs associated with them (Allen 2003). Recently, biofortification has been introduced as a cost-effective complementary strategy of dealing with micronutrient deficiencies such as VAD (De Groote \& Kimenju, 2008; Domonko et al., 2018).

Biofortification, which deals with breeding staple crops for higher micronutrients could be done using both traditional and genetically modified breeding techniques. For example, in the HarvestPlus challenge program of the Consultative Group on International Agricultural research, plant breeders have developed crops with high levels of micronutrient using traditional breeding techniques (De Groote \& Kimenju, 2008). Currently, biofortified crop varieties of sweet potato, cassava and maize have been released on the market. Also, high vitamin A fortified rice called "Golden rice" has been developed with high potential effect of dealing with VAD (Zimmermann \& Qaim, 2004).

Previous studies have examined consumer preferences for biofortified foods (De Groote \& Kimenju, 2008; Domonko et al., 2018; González, Johnson, \& Qaim, 2009; Pambo, Otieno, \& Okello, 2017; Zimmermann \& Qaim, 2004) using different techniques. For instance, González et al. (2009) examined consumer acceptance of biofortified cassava in 
Brazil using contingent valuation and contingent ranking techniques and found that consumers preferred affordable, GM-free and white cassava. Pambo et al. (2017) study on consumer preferences for vitamin A fortified sugar revealed that consumers have heterogenous preferences for key attributes such as fortification labels, and minimum levels of vitamin A and color of the grains. In Domonko et al. (2018) study on consumer preferences for vitamin A biofortified rice in Tanzania, the authors found that consumers preferred rice that decreased the risk of visual impairment, is white in color with longer grains.

Although previous studies have revealed that understanding the factors that drive consumer demand for biofortified foods would prove useful in the design and implementation of programmes aimed at addressing VAD, not many of those studies have been conducted in the Ghanaian context. The only study conducted relate to consumer preferences for orange fleshed sweet potato (Etumnu, 2016) and biofortified maize (De Groote et al., 2010). Rice, which is the second most consumed food in both the urban and rural areas have not been examined to ascertain consumer preferences for its biofortification.

This study is therefore the first empirical study on consumer preferences for biofortified rice in Ghana. Specifically, it examines consumer preferences for different attributes of biofortified rice. We need information on consumers' valuation of vision characteristics of the biofortified rice (whether it reduces blindness by half or reduces blindness completely). It is also of interest for consumers to evaluate the color of the rice (white, brown and yellow), evaluate the length (small, medium and long), and the price attribute, which would be used to evaluate the willingness to pay. The problem is examined using a stated preference approach.

Stated preference approach is a non-market valuation technique that relies on answers to carefully worded survey questions in the form of amounts, choices, ratings and indication of preferences (Brown, 2003). Stated preference method is mainly of two types: contingent valuation and discrete choice experiment. In this paper, the discrete choice approach is adopted because of its capability of estimating accurate willingness to pay values and also valuing goods or services with multiple attributes. Discrete choice experiment is an established method that is used to identify behavioral drivers of choice where consumers are presented with hypothetical scenarios and asked to make choices between them (Owusu Coffie et al. 2016).

In this study, recent advances in the choice experiment literature such as the generalized multinomial logit (GMNL) model of Fiebig et al. (2010) and the mixed logit (MIXL) model of Greene \& Hensher (2010) have been employed. In addition, to examine consumers' willingness to pay (WTP) for the attributes of the biofortified rice, willingness to pay space model within the GMNL and MIXL frameworks have been adopted (Hole \& Kolstad, 2012; Owusu Coffie et al., 2016). The findings would have greater implications for the fortification program of the Government of Ghana, and also for breeding stations involved in developing biofortified rice.

The data for empirical application come from a choice experiment study conducted in Ghana among 153 households from selected communities in the Central region of Ghana. The econometric modeling revealed that, on average, consumers have preferences for biofortified rice with vision attribute that either reduces blindness by half or completely removes blindness, although the utility attached to removing blindness completely is higher. Such a biofortified rice preferably should be white in color and cheap with small grain sizes. Furthermore, the findings revealed that preferences for biofortified rice are different across gender groups such as the young, males and females and these variations have to be considered in breeding biofortified rice for consumers.

The rest of the paper is structured as follows. The next section describes review of related literature. This is followed by the methodology comprising theoretical and econometric frameworks, choice experiment design and data used for empirical application. Empirical results and discussion are presented next and the paper finally concludes with policy implications. 


\section{LITERATURE REVIEW}

Rice is a commodity of strategic importance to Africa and has become the fastest growing food source to both rural and urban dwellers (Nwanze, Mohapatra, Kormawa, Keya, \& Bruce-Oliver, 2006; Quaye, Johnson-Kanda, Gayin, \& Manful, 2010). In many countries in Africa, the crop is gradually replacing traditional foods, mainly roots and tubers. In Ghana, for example, it is an important cash crop that forms an integral part of the Ghanaian diet, and is essential in ensuring food security among rural and urban households (Owusu Coffie et al., 2016). The per capita demand for rice in Ghana is about $68 \mathrm{~kg}$ per year. Current rice demand outstrips supply and the deficit in supply is filled mainly through imports.

Although rice has become an important food crop in Ghana, it lacks some vital nutrients especially micronutrients. Micronutrients are very essential to maintain the health of both adult and children. Inadequate supply of micronutrients often referred to as hidden hunger is a very serious problem in many developing countries. Fighting micronutrient malnutrition is an integral part of the millennium development goals and now the sustainable development goals (Allen, 2000). Over the years, micronutrient malnutrition has been addressed through preventive and curative intervention measures (Mayer, Pfeiffer, \& Beyer, 2008), often referred to as industrial fortification. Vitamin $A$ is one of the micronutrients that has received lots of attention with industrial fortification, mainly because of high rate of deficiency and mortality associated with it (De Groote et al., 2010). The industrial fortification interventions, although have been very beneficial are expensive in implementation. Biofortification has therefore been suggested as a cost effective approach to augmenting industrial fortification projects.

Biofortification of crops has been developed with increased bioavailable concentrations of essential micronutrients (Mayer et al., 2008). Since the introduction of biofortified crops, a number studies have examined consumer preferences for such crops in combating vitamin $A$ deficiency (De Groote et al., 2010; Domonko et al., 2018; González et al., 2009). For example, Chowdhury et al. (2011) examined consumers' willingness to pay for biofortified foods in Uganda using a choice experiment approach. The study found that taste is very important in consumer acceptance and willingness to pay for biofortified foods. In a similar study, De Groote et al. (2011) conducted a study into consumer willingness to pay for food quality with experimental auctions and found that consumers were willing to pay higher premiums for biofortified maize compared to yellow maize. Other studies include González et al. (2009), Pambo et al. (2017) and Domonko et al. (2018).

\section{METHODS OF RESEARCH}

Economic valuation of hypothetical goods could be achieved using either revealed preference approach or stated preference approach. The revealed preference approach is used to examine product demand by examining actual purchases of related goods in the private market (Pambo et al., 2017). The stated preference approach, which includes contingent valuation and choice modelling on the other hand, evaluates consumers preferences for hypothetical scenarios (Pambo et al., 2017). In contingent valuation, respondents are led to state their maximum willingness to pay or willingness to accept compensation for a hypothetical change in an environment or economic good (Mitchell \& Carson, 1989; Pambo et al., 2017). The choice modelling enables consumers to select multiple attributes of a product or service and their trade-offs simultaneously.

Choice modelling is consistent with the theory of consumer behavior and therefore used to analyze consumer choice behavior based on the random utility theory of McFadden (1974). It draws from Lancaster's characteristic theory of value, which states that consumers derive utility from the characteristics of a good rather than the good itself (Lancaster, 1966). Its econometric basis is the random utility theory of Manski (1977), which states that utility is unobservable but can be measured as the probability that rational consumers will choose goods, in a given choice set from which they obtain the highest utility. Although there are a number of choice modelling approaches, choice experiment is the most consistent with 
welfare estimation (Alphonce \& Alfnes, 2012; Birol, Karousakis, \& Koundouri, 2006) because it enables one to estimate trade-offs and values of the attributes of a good and provides useful information for strategic planning and interventions (Hanley, Mourato, \& Wright, 2001).

The attributes of alternative $j$ in choice occasion $t$ faced by respondent $n$ could be labelled as vector $X_{n j t}$. The utility obtained by individual $n$ from alternative $j$ in choice occasion $t$ is specified as:

$$
U_{n j t}=\beta_{n} X_{n j t}+\varepsilon_{n j t}
$$

Where: the coefficients of $\beta_{n}$ is unobserved and varies in the population with a density function $f\left(\beta_{n} / \theta\right)$ while $\theta$ are parameters to be estimated. $\varepsilon_{n j t}$ is an unobserved random term that is identically and independently distributed. The unconditional probability of the sequence of choices made by an individual is expressed as:

$$
P_{n j t}(\theta)=\int L_{n j}\left(\beta_{n}\right) f\left(\beta_{n} / \theta\right) d \beta_{n}
$$

The mixed logit specified in Eq. (2) accounts for only unconditional heterogeneity but not conditional heterogeneity (explain the sources of heterogeneity). To account for conditional heterogeneity, model expansion is required to incorporate socio-economic characteristics of respondents. This process enables the model to pick up both random and conditional heterogeneity and further improves the model fit (Birol et al. 2006). Including respondents' socio-economic characteristics as $S_{n}$, results in Eq. (3):

$$
P_{n j t}=\int \frac{e^{\beta_{n}^{\prime} x_{n j}}+S_{n}}{\sum_{j=1}^{j} e^{\beta_{n}^{x} x j}+S_{n}} f\left(\beta_{n} / \theta\right) d \beta_{n}
$$

Where: all parameters are as earlier defined. In this paper, the mixed logit model is extended into a generalized multinomial logit model (GMNL). The GMNL model was developed by Fiebig et al. (2010) and Greene (2012) to account for both scale heterogeneity (choice inconsistency) and preference heterogeneity. In the GMNL model, the utility of individual $n$ choosing from alternative $j$ on choice occasion $t$ is given as

$$
U_{n j t}=\left[\sigma_{n} \beta+\gamma \eta_{n}+(1-\gamma) \sigma_{n} \eta_{n}\right] x_{n j t}+\varepsilon_{n j t}
$$

Where: $\gamma$ is a scale parameter between 0 and 1. The scale effect on the individual idiosyncratic component of taste is composed of two parts: unscaled idiosyncratic effect $\left(\gamma \eta_{n}\right)$ and scaled idiosyncratic effect $(1-\gamma)$. Several models could be formed based on restrictions on the parameters (see Fiebig et al. (2010) for details). Specifically, we have GMNL-I and GMNL-II. The difference between GMNL-I and GMNL-II is that in GMNL-I, the standard deviation $\gamma \eta_{n}$ is independent of the scaling of $\beta$, whereas in -MNL-II, it is proportional to the scale heterogeneity $(\sigma)$ (Kassie et al., 2017). GMNL approaches GMNL-I as $\mathrm{Y}$ approaches 1 , and it approaches GMNL-II as $\mathrm{y}$ approaches 0 . In this paper, the GMNLII was adopted in the estimation of both scale and preference heterogeneity.

Maximum likelihood (ML) is often employed in accounting for preference heterogeneity. The ML works on the principle of searching for a solution by simulating $n$ draws from distributions with given means and standard deviations (Birol et al., 2006). Joint simulated distribution integration is used to obtain probabilities. The standard approach to simulation estimation is based on random draws. However, with large samples and complex models, this can be very time consuming. The Halton draw, therefore serve as an alternative to the random draws with the advantage of speed gains and no degradation in simulation performance (Revelt \&Train 1998).

The consistency of choice experiment with consumer theory makes it suitable in estimating welfare effects such as the willingness to pay. Willingness to pay (WTP) is a 
measure of the trade-off between non-price attributes and a price attribute. In the choice experiment literature, two approaches have been proposed in estimating WTP: the indirect ratio method and the direct method (Owusu Coffie et al., 2016). The indirect approach, also called preference space model has received lots of criticisms in the literature because of the biases it introduces into the WTP values (Hole \& Kolstad, 2012). In this paper, the direct approach, which is simply a re-parameterization of the model to estimate the taste parameters in WTP space is adopted.

The WTP within the preference space given a distinction between the price, $P_{n j t}$, and non-price attributes, $x_{n j t}$, could be specified as:

$$
U_{n j t}=\beta_{n p} P_{n j t}+\sum_{i=1}^{i} \beta_{n j} x_{n j t}
$$

Where: $\beta_{n p}$ is the coefficient of the price attribute and $\beta_{n j}$ is a vector of the coefficients of the non-price attributes.

The WTP space model requires a reformulation of Eq. (5) so that direct coefficients would be obtained as WTP values (Owusu Coffie et al., 2016), and this is given as:

$$
U_{n j t}=\beta_{n p}\left[P_{n j t}+\sum_{i=1}^{i} \beta_{n j}^{*} x_{n j t}\right]+\varepsilon_{n j t}
$$

Where: $\beta_{n j}^{*}=\beta_{n j} / \beta_{n p}$ and $\beta_{n p}$ is a normalizing constant; $\beta_{n j}^{*}$ is a vector of WTP values obtained from Eq. (6).

The starting point of discrete choice experiment modelling is to identify attributes and levels. Based on literature search and experts' interview, the following attributes were identified: vision benefit, color of the fortified rice, length of the fortified rice and price per $\mathrm{kg}$. The vision attribute was of three levels and these were; does not decrease the incidence of blindness, reduces the incidence of blindness by half and eliminates the incident of blindness. There were also three levels to the color attribute (white, brown and yellow). The length and the price attributes were of three levels each: small, medium and long for the length attribute and price (6 GHS, 12 GHS and 18 GHS).

Upon the identification of the attributes and their corresponding levels, an efficient choice experiment design was generated in STATA 14 with priors obtained from a pilot study conducted in the University of Cape Coast environs. Efficient design is more suitable because it is cost effective and increases sampling (Bliemer and Rose 2010). The final design had 30 paired choice sets that were randomly grouped into 10 scenarios. Each choice set was composed of two alternatives (A and B), and a third alternative, that represented none of the options. The attributes, descriptions and levels are presented in Table 1.

\begin{tabular}{|c|c|c|c|}
\hline Attributes & Descriptions & Levels & Reference level \\
\hline $\begin{array}{l}\text { Vision } \\
\text { benefit }\end{array}$ & $\begin{array}{l}\text { Vision benefit for the consumption of } \\
\text { biofortified rice }\end{array}$ & $\begin{array}{l}\text { Does not decrease blindness or night } \\
\text { blindness } \\
\text { Reduces the level of both blindness } \\
\text { and night blindness by half } \\
\text { Removes completely blindness and } \\
\text { night blindness }\end{array}$ & $\begin{array}{l}\text { Does not decrease blindness } \\
\text { or night blindness }\end{array}$ \\
\hline Color & Color of the biofortified rice & $\begin{array}{l}\text { White } \\
\text { Brown } \\
\text { Yellow }\end{array}$ & White \\
\hline Length & Length of the biofortified rice & $\begin{array}{l}\text { Small } \\
\text { Medium } \\
\text { Long }\end{array}$ & Small \\
\hline Price/kg & $\begin{array}{l}\text { Monetary attribute: price of } 1 \mathrm{~kg} \text { of } \\
\text { fortified rice in Ghana }\end{array}$ & $\begin{array}{l}6 \mathrm{GHS} \\
12 \mathrm{GHS} \\
18 \mathrm{GHS}\end{array}$ & \\
\hline
\end{tabular}

Table 1 - Attribute description

Note: at the time of the survey, 1 GHS $=5$ USD. 
The target population included consumers in the Central region of Ghana. Respondents were selected using a multistage sampling technique. In the first stage, Cape Coast metropolis, Anomabo and Agona Sweduro suburbs of the Central region of Ghana were selected. This was followed by the selection of two communities each from the selected districts. Household heads responsible for food purchase in the house were interviewed. The overall sample for the study comprised 153 households, with 50 each from Cape Coast and Anomabo and 53 from Agona Sweduro. The data collection, which stated in May 2019, lasted for about a month. The survey comprised information on the socio-economic characteristics of the respondents and also the choice experiment. Each respondent was asked to choose his/her preferred biofortified rice alternative in 10 choice situations. Table 2 shows an example of choice set.

Table 2 - Sample choice set

\begin{tabular}{|c|c|c|c|}
\hline Attributes & Option A & Option B & Option C \\
\hline Vision & $\begin{array}{l}\text { Does not decrease risk of } \\
\text { blindness }\end{array}$ & $\begin{array}{l}\text { Completely decrease risk } \\
\text { of blindness }\end{array}$ & \multirow[t]{4}{*}{ Opt out } \\
\hline Color & White & Brown & \\
\hline Length & Small & Long & \\
\hline Purchase Price (kg) & GHS18.00 & GHS18.00 & \\
\hline I would prefer to buy & & & \\
\hline
\end{tabular}

The socio-economic characteristics of the sample are presented in Table 3. From the table, the average age of the sample is 28 years, indicating a fairly youthful population. For sex, the mean of 0.37 indicates that males mainly dominate the sample given that the sex variable is coded as zero (0) for males and one (1) for females. The vitamin A deficiency (VAD) awareness variable is about $97 \%$ showing that majority of the sample are aware of vitamin A deficiency or at least have heard about VAD. The risk of VAD was measured on a Likert scale where 1=no risk, 2=little risk, 3=neither low nor high risk, 4=medium risk, 5= serious risk. Given an average of almost 4 gives an indication of respondents perceived risk of VAD. The risk of blindness also measured on the same scale as VAD shows that respondents perceived night blindness as a serious risk. Considering the choices of the biofortified rice scenarios, it is observed from the table that choices where equally distributed among the three alternatives.

Table 3 - Sample socio-economic characteristics

\begin{tabular}{lll}
\hline $\mathrm{n} / \mathrm{n}$ & Mean & SD \\
\hline Socio-economics & 28 & 8 \\
Age & 0.37 & 0.48 \\
Sex & 0.97 & 0.16 \\
VAD awareness & 3.53 & 1.063 \\
Risk of vitamin A deficiency $^{\text {a }}$ & 3.62 & 1.042 \\
Risk of night blindness $^{{ }^{2}}$ & Percentage & \\
Choices $_{\text {Option A }}$ & $33.3 \%$ & \\
Option B & $33.3 \%$ & \\
Option C & $33.3 \%$ & \\
\hline
\end{tabular}

Note: were measured on a Likert scale where 1=no risk, 2=little risk, 3=neither low nor high risk, 4=medium risk, $5=$ serious risk.

\section{RESULTS AND DISCUSSION}

The results of the mixed logit and the generalized multinomial logit models are presented in Table 4. From the table, except for the medium grain attribute, all the other coefficients in the MIXL model are significant. Specifically, the results show that consumers hold a positive utility for biofortified rice with vision attribute that reduces blindness or completely removes blindness. They also have utility of biofortified rice that is white in color 
as opposed to brown or yellow. This finding is consistent with Domonko et al. (2018) study on the preferences of consumers in Tanzania.

The long grain attribute is negative and significant indicating that consumers would rather prefer short grain biofortified rice and are indifferent towards medium grain biofortified rice. This finding is contrary to Domonko et al. (2018) who found that consumers prefer long grain rice to short or medium grain rice. The price coefficient is also negative and significant showing that consumers would prefer cheap biofortified rice as opposed to expensive ones. Once again this finding confirms Domonko et al. (2018) study outcome. Similar to the MIXL model estimates, all the coefficients of the GMNL model are significant except for medium grain attribute (Table 4). All the standard deviations of the MIXL model are significant showing that there are unobserved heterogeneity in the sample.

Table 4 - Results of the MIXL and GMNL models

\begin{tabular}{|c|c|c|c|c|}
\hline & \multicolumn{2}{|c|}{ Model 1=MIXL } & \multicolumn{2}{|c|}{ Model 2=GMNL } \\
\hline & Mean SE & & Mean & SE \\
\hline \multicolumn{5}{|l|}{ Taste parameters } \\
\hline Reduces blindness by half & $2.830^{\star * *}$ & 0.275 & $5.757^{* * *}$ & 0.924 \\
\hline Completely removes blindness & $6.274^{\star * *}$ & 0.617 & $10.714^{\star * *}$ & 1.692 \\
\hline Brown color & $-1.355^{\star * \star}$ & 0.256 & $-2.837^{\star \star \star}$ & 0.713 \\
\hline Yellow color & $-0.728^{\star \star \star}$ & 0.199 & $-1.986^{\star \star \star}$ & 0.544 \\
\hline Medium grain & 0.060 & 0.165 & -0.201 & 0.394 \\
\hline Long grain & $-0.369^{* *}$ & 0.168 & $-1.171^{\star *}$ & 0.401 \\
\hline Price & $-0.733^{\star *}$ & 0.259 & $-1.527^{\star \star}$ & 0.475 \\
\hline Status quo & $-0.870^{*}$ & 0.524 & $-2.387^{\star *}$ & 0.998 \\
\hline Tau & - & - & $1.274^{\star \star \star}$ & 0.117 \\
\hline \multicolumn{5}{|l|}{ Heterogeneity in mean } \\
\hline Reduces blindness by half & $1.729^{\star * *}$ & 0.307 & -0.067 & 0.766 \\
\hline Completely removes blindness & $3.488^{\star * *}$ & 0.479 & -0.201 & 0.783 \\
\hline Brown color & $1.405^{\star * *}$ & 0.412 & $2.220^{* *}$ & 0.702 \\
\hline Yellow color & $0.991^{* * *}$ & 0.270 & $1.130^{* *}$ & 0.481 \\
\hline Medium grain & -0.307 & 0.304 & 0.086 & 0.469 \\
\hline Long grain & $0.571^{\star *}$ & 0.242 & 0.237 & 0.621 \\
\hline Price & -0.260 & 0.245 & $-0.864^{* *}$ & 0.421 \\
\hline Status quo & $4.880^{\star * *}$ & 0.524 & $7.827^{* \star}$ & 1.286 \\
\hline LL & \multirow{3}{*}{\multicolumn{2}{|c|}{$\begin{array}{l}-839.761 \\
1814.417 \\
4587\end{array}$}} & \multirow{3}{*}{\multicolumn{2}{|c|}{$\begin{array}{l}-807.394 \\
1749.68 \\
4587\end{array}$}} \\
\hline BIC & & & & \\
\hline $\mathrm{N}$ & & & & \\
\hline
\end{tabular}

Note: $S E=$ Standard errors, $L L=L$ og likelihood, $N=$ Number of observations.

The advanced discrete choice experiment models although address the problems of unobserved heterogeneity, fail to explain the sources of heterogeneity (Boxall \& Adamowicz 2002). Conditional heterogeneity deals with accounting for heterogeneity conditioned on individual specific characteristics (Birol et al. 2006). In this paper, conditional heterogeneity was accounted for some socio-economic variables (age, gender and consumers perceived level of risk of VAD) with the attributes within the MIXL and GMNL model framework. The model estimates are reported in Table 5.

From the estimated coefficients in the table, respondents with high level of VAD risk associate high utility with biofortified rice with vision attribute that reduces blindness or completely removes blindness, although the level of utility attached to the vision attribute that completely removes blindness is more than twice (2.96) that of the utility associated with reducing blindness by half (1.25). Similarly those who associate high perceived level of risk associated with VAD are indifferent towards brown rice but have a negative utility towards yellow rice. In addition, the aged associate higher utility with biofortified rice with vision attribute that reduces blindness by half or completely removes blindness. This finding is not surprising given that eye health reduces with age (Chader \& Taylor, 2013). They however, prefer biofortified rice with white color compared to brown or yellow color. 
Also, the aged are indifferent towards medium grains but hold disutility for long grains. Furthermore, females prefer biofortified rice with brown or yellow color but the preference for the brown rice is higher. Having accounted for heterogeneity, it is observed that sampled consumers prefer both brown and yellow rice as opposed to white rice, suggesting that accounting for both observed and unobserved heterogeneity is important for robust findings with conclusive policy recommendations.

The willingness to pay measures the substitution between a monetary attribute and a non-monetary attribute. In this paper, the willingness to pay space model was adopted to estimate the willingness to pay for the biofortified rice attributes because of the problems associated with the conventional ratio method of estimating the willingness to pay (Hole \& Kolstad, 2012).

From the estimated results (Table 6), implicit prices of biofortified rice with vision attribute that reduces blindness by half and completely removes blindness are higher compared to other attributes. This finding is relevant because Ghana has a very high incidence of vitamin A deficiency (Taylor 2014), close to $76 \%$. Therefore, it does not come as a surprise with consumers' value attached to the vision attribute of the proposed biofortified rice.

Table 5 - Results of the Heterogeneity in mean parameters models

\begin{tabular}{|c|c|c|c|c|}
\hline & \multicolumn{2}{|c|}{ Model 3=MIXL } & \multicolumn{2}{|c|}{ Model 4=GMNL } \\
\hline & Coef. & SE & Coef. & SE \\
\hline \multicolumn{5}{|l|}{ Taste parameters } \\
\hline Reduces blindness by half & -0.074 & 0.769 & 2.446 & 1.731 \\
\hline Completely removes blindness & -1.252 & 1.315 & 2.478 & 2.525 \\
\hline Brown color & $2.549^{\star *}$ & 1.026 & 2.212 & 1.649 \\
\hline Yellow color & $1.469^{\star *}$ & 0.748 & -1.168 & 1.422 \\
\hline Medium grain & 1.030 & 0.691 & 1.806 & 1.098 \\
\hline Long grain & $1.255^{\star}$ & 0.717 & 1.814 & 1.230 \\
\hline Price & $-0.726^{\star * *}$ & 0.269 & $-0.672^{* *}$ & 0.264 \\
\hline Status quo & $-2.435^{\star \star \star}$ & 0.577 & $-2.187^{\star *}$ & 0.804 \\
\hline \multicolumn{5}{|l|}{ Observed heterogeneity } \\
\hline Reduces blindness by half* Risk of VAD & $1.248^{\star * \star}$ & 0.393 & $3.583^{\star \star *}$ & 0.818 \\
\hline Completely removes blindness ${ }^{*}$ Risk of VAD & $2.956^{\star \star \star}$ & 0.689 & $6.392^{\star \star \star}$ & 1.257 \\
\hline Brown color ${ }^{\star}$ Risk of VAD & -0.151 & 0.469 & $-1.662^{\star *}$ & 0.766 \\
\hline Yellow color* Risk of VAD & $-0.891^{* *}$ & 0.370 & $-2.191^{* * *}$ & 0.762 \\
\hline Medium grain* Risk of VAD & 0.236 & 0.335 & 0.463 & 0.607 \\
\hline Long grain ${ }^{\star}$ Risk of VAD & 0.141 & 0.344 & -0.498 & 0.653 \\
\hline Reduces blindness by half*Age & $0.090^{* * *}$ & 0.030 & 0.014 & 0.068 \\
\hline Completely removes blindness ${ }^{*} \mathrm{Age}$ & $0.190^{\star * *}$ & 0.052 & 0.082 & 0.105 \\
\hline Brown color ${ }^{\star}$ Age & $-0.139^{* \star *}$ & 0.040 & $-0.121^{*}$ & 0.067 \\
\hline Yellow color*Age & $-0.072^{\star *}$ & 0.029 & -0.060 & 0.056 \\
\hline Medium grain*Age & -0.031 & 0.027 & -0.059 & 0.043 \\
\hline Long grain*Age & $-0.059^{* *}$ & 0.029 & -0.078 & 0.050 \\
\hline Reduces blindness by half** Sex & -0.381 & 0.390 & 0.379 & 0.517 \\
\hline Completely removes blindness ${ }^{*}$ Sex & 0.748 & 0.626 & 1.265 & 1.026 \\
\hline Brown color ${ }^{\star}$ Sex & -0.467 & 0.471 & -0.271 & 0.580 \\
\hline Yellow color ${ }^{\star}$ Sex & $0.042^{*}$ & 0.357 & 0.140 & 0.492 \\
\hline Medium grain*Sex & -0.629 & 0.334 & -0.610 & 0.477 \\
\hline Long grain* Sex & -0.259 & 0.319 & -0.030 & 0.452 \\
\hline \multicolumn{5}{|l|}{ Heterogeneity in mean } \\
\hline Reduces blindness by half & $1.311^{* \star *}$ & 0.216 & $-0.378^{* *}$ & 0.165 \\
\hline Completely removes blindness & $3.144^{\star * *}$ & 0.506 & -0.276 & 0.423 \\
\hline Brown color & $1.185^{\star \star \star}$ & 0.281 & $2.050^{\star \star *}$ & 0.379 \\
\hline Yellow color & $-0.735^{\star \star \star}$ & 0.239 & 0.555 & 0.338 \\
\hline Medium grain & -0.011 & 0.234 & $0.375^{\star}$ & 0.215 \\
\hline Long grain & 0.297 & 0.254 & 0.268 & 0.246 \\
\hline Price & $-0.499^{* \star \star}$ & 0.158 & $-0.928^{\star * \star}$ & 0.313 \\
\hline Status quo & $7.115^{* * *}$ & 0.767 & $8.840^{\star * *}$ & 1.156 \\
\hline Tau & & & $1.046^{\star \star \star}$ & 0.079 \\
\hline LL & -807.199 & & -778.799 & \\
\hline $\mathrm{BIC}$ & 1901.051 & & 1852.683 & \\
\hline $\mathrm{N}$ & 4587 & & 4587 & \\
\hline
\end{tabular}

Note: $S E=$ Standard errors, $L L=L$ og likelihood, $N=$ Number of observations. 
Table 6 - Willingness to pay for biofortified rice in WTP space

\begin{tabular}{|c|c|c|c|c|}
\hline & \multicolumn{2}{|c|}{ Model 5=GMNL } & \multicolumn{2}{|c|}{ Model 6=GMNL } \\
\hline & Mean & SE & Coef. & SE \\
\hline \multicolumn{5}{|l|}{ Taste parameters } \\
\hline Reduces blindness by half & $-3.283^{* * *}$ & 0.619 & $-2.297^{\star \star \star}$ & 0.393 \\
\hline Completely removes blindness & $-5.959^{\star \star \star}$ & 1.161 & $-3.382^{\star \star *}$ & 0.749 \\
\hline Brown color & $1.577^{* \star *}$ & 0.359 & $-0.683^{*}$ & 0.365 \\
\hline Yellow color & $1.069^{\star * *}$ & 0.284 & $-0.925^{\star \star}$ & 0.389 \\
\hline Medium grain & -0.069 & 0.208 & 0.499 & 0.366 \\
\hline Long grain & $0.650^{* *}$ & 0.259 & $-1.134^{\star \star *}$ & 0.379 \\
\hline Price & 1 & Fixed & 1 & Fixed \\
\hline Status quo & $-2.344^{\star \star *}$ & 0.519 & $-1.954^{\star \star \star}$ & -0.526 \\
\hline Constant & $0.544^{* * *}$ & 0.103 & $2.078^{\star * \star}$ & 0.243 \\
\hline Tau & $1.340^{* * *}$ & $0.155^{\star * *}$ & $1.986^{\star * *}$ & 0.141 \\
\hline \multicolumn{5}{|l|}{ Observed heterogeneity } \\
\hline Reduces blindness by half* Risk of VAD & - & - & -0.054 & 0.191 \\
\hline Completely removes blindness* Risk of VAD & - & - & $-0.605^{\star}$ & 0.345 \\
\hline Brown *Risk of VAD & - & - & $-0.378^{* *}$ & 0.176 \\
\hline Yellow color ${ }^{\star}$ Risk of VAD & - & - & $-1.304^{\star * *}$ & 0.243 \\
\hline Medium grain* Risk of VAD & - & - & 0.455 & 0.219 \\
\hline Long grain*Risk of VAD & - & - & -0.151 & 0.195 \\
\hline Reduces blindness by half*Age & - & - & -0.001 & 0.012 \\
\hline Completely removes blindness*Age & - & - & 0.036 & 0.223 \\
\hline Brown color*Age & - & - & $-0.044^{* * *}$ & 0.013 \\
\hline Yellow color*Age & - & - & -0.017 & 0.014 \\
\hline Medium grain*Age & - & - & $-0.028^{\star *}$ & 0.011 \\
\hline Long grain*Age & - & - & $-0.040^{* *}$ & 0.012 \\
\hline Reduces blindness by half ${ }^{\star \star}$ Sex & - & - & 0.136 & 0.187 \\
\hline Completely removes blindness*Sex & - & - & $0.696^{\star *}$ & 0.327 \\
\hline Brown color*Sex & - & - & -0.224 & 0.180 \\
\hline Yellow color*Sex & - & - & -0.278 & 0.259 \\
\hline Medium grain*Sex & - & - & $-0.394^{*}$ & 0.227 \\
\hline Long grain*Sex & - & - & $-0.536^{\star *}$ & 0.219 \\
\hline \multicolumn{5}{|l|}{ Heterogeneity in mean } \\
\hline Reduces blindness by half & $0.667^{\star * *}$ & 0.178 & $1.059^{\star * *}$ & 0.107 \\
\hline Completely removes blindness & $-0.694^{\star \star \star}$ & 0.261 & $-1.301^{* * *}$ & 0.184 \\
\hline Brown color & $-1.346^{\star *}$ & 0.308 & $1.181^{* * *}$ & 0.133 \\
\hline Yellow color & 0.025 & 0.242 & $0.902^{\star \star \star}$ & 0.102 \\
\hline Medium grain & 0.152 & 0.163 & -0.086 & 0.068 \\
\hline Long grain & $-0.667^{*}$ & 0.348 & -0.027 & 0.075 \\
\hline Price & - & - & - & - \\
\hline Status quo & $9.017^{* * *}$ & 1.159 & $6.622^{\star * *}$ & $1-153$ \\
\hline LL & -808.213 & & -796.469 & \\
\hline
\end{tabular}

Note: SE=Standard errors, $L L=L o g$ likelihood.

Considering the WTP estimates without heterogeneity, sampled consumers are willing to pay a premium for vision attribute associated with completely removing blindness that is about 2.98 times and 3.66 times the amount they are willing to pay for long grain and yellow color attributes, respectively. Also sampled consumers are willing to pay a premium for vision attribute that completely removes blindness that is 1.47 times the amount they are willing to pay for a vision attribute that removes blindness by half. Similarly, sampled consumers are willing to pay a premium for yellow colored biofortified rice that is 1.35 times the amount they are willing to pay for brown colored biofortified rice. Considering heterogeneity in the mean willingness to pay estimates, sampled young consumers are willing to pay for rice with brown color and have medium or long grains as opposed to rice with white color with short grains. Females are also willing to pay for rice that completely removes blindness compared to rice that does not remove blindness. Males on the other hand are willing to pay for biofortified rice that has either medium or long grains compared with small grains. 


\section{CONCLUSION}

Biofortification of staple foods such as rice serves as a cheap strategy to addressing the problem of micronutrient malnutrition including vitamin A deficiency (VAD) in developing countries. In this paper, consumer preferences for biofortified rice were examined using recent advances in the choice experiment literature. Specifically, the mixed logit model and generalized multinomial logit models were used to examine Ghanaian consumer preferences for biofortified rice. In addition, the willingness to pay space model was used to estimate the substitution between the multiple attributes of the biofortified rice.

Using a sample of 153 , the econometric modelling revealed, on average, sampled consumers prefer biofortified rice with vision attribute that either reduces blindness by half or completely removes blindness. However, they value the vision attribute that completely removes night blindness above that which would reduce night blindness by half. This finding is consistent with Domonko et al. (2018) study outcome on consumer preferences for biofortified rice in Tanzania. The results also show that the sampled consumers prefer white colored rice with short grains compared to brown or yellow colored rice with medium or long grains. This finding corroborates similar studies on other food produce such as maize that has revealed that generally consumers have preferences for white colored food produce (De Groote \& Kimenju, 2008), and so are other surveys conducted (Rubey, Ward, \& Tschirley, 1997; Tschirley \& Santos, 1995).

In addition, it was found that sampled female consumers are willing to pay for biofortified rice that completely removes night blindness. The males are rather willing to pay for rice that has either medium or long grains compared with small grains. The findings therefore show that there is heterogeneity in the preferences of consumers for the biofortified rice grains, however, they are willing to purchase biofortified rice that is affordable with short grains and completely removes night blindness.

The findings of this study have several policy implications. Consumers' preference for white colored rice has implications for breeding purposes, and successful implementation of programs aimed at fighting micronutrient deficiency, especially vitamin A. For instance, there would be the need to create awareness among consumers to increase patronage for biofortified food produce because they are likely to have different color other than white. Also, discounts would have to be provided to consumers for the purchase of biofortified food produce that are colored, (De Groote et al., 2011) to promote consumption. In addition, variation in preferences across gender groups such as the young, males and females should be considered in breeding biofortified rice for consumers.

\section{REFERENCES}

1. Allen, L. H. (2000). Ending hidden hunger: The history of micronutrient deficiency control. Background Analysis for the World Bank-UNICEF Nutrition Assessment Project World Bank.

2. Alphonce, R., \& Alfnes, F. (2012). Consumer willingness to pay for food safety in Tanzania: An incentive-aligned conjoint analysis. International Journal of Consumer Studies, 36(4), 394-400.

3. Birol, E., Karousakis, K., \& Koundouri, P. (2006). Using a choice experiment to account for preference heterogeneity in wetland attributes: The case of Cheimaditida wetland in Greece. Ecological Economics, 60(1), 145-156.

4. Brown, T. C. (2003). Introduction to stated preference methods. In A primer on nonmarket valuation (pp. 99-110). Springer.

5. Chader, G. J., \& Taylor, A. (2013). Preface: The aging eye: normal changes, age-related diseases, and sight-saving approaches. Investigative Ophthalmology \& Visual Science, 54(14), ORSF1-ORSF4.

6. Chowdhury, S. (2011). Are consumers in developing countries willing to pay more for micronutrient-dense biofortified foods? Evidence from a field experiment in Uganda. American Journal of Agricultural Economics, 93(1), 83-97. 
7. Christian, P., Bunjun Srihari, S., Thorne-Lyman, A., Khatry, S. K., LeClerq, S. C., \& Ram Shrestha, S. (2006). Eating down in pregnancy: Exploring food-related beliefs and practices of pregnancy in rural Nepal. Ecology of Food and Nutrition, 45(4), 253-278.

8. De Groote, H., \& Kimenju, S. C. (2008). Comparing consumer preferences for color and nutritional quality in maize: Application of a semi-double-bound logistic model on urban consumers in Kenya. Food Policy, 33(4), 362-370.

9. De Groote, H., Kimenju, S. C., \& Morawetz, U. B. (2011). Estimating consumer willingness to pay for food quality with experimental auctions: The case of yellow versus fortified maize meal in Kenya. Agricultural Economics, 42(1), 1-16.

10. De Groote, H., Tomlins, K., Haleegoah, J., Awool, M., Frimpong, B., Banerji, A., ... Meenakshi, J. V. (2010). Assessing rural consumers' WTP for orange, biofortified maize in Ghana with experimental auctions and a simulated radio message.

11. Domonko, E. S. (2018). Consumer risk perception of vitamin A deficiency and acceptance of biofortified rice in the Morogoro region of Tanzania. African Journal of Agricultural and Resource Economics, 13(311-2018-2939), 1-14.

12. Etumnu, C. (2016). Behavioral Determinants of Biofortified Food Acceptance: The Case of Orange-fleshed Sweet Potato in Ghana.

13. González, C., Johnson, N., \& Qaim, M. (2009). Consumer acceptance of secondgeneration GM Foods: The case of biofortified cassava in the North-east of Brazil. Journal of Agricultural Economics, 60(3), 604-624.

14. Hanley, N., Mourato, S., \& Wright, R. E. (2001). Choice modelling approaches: A superior alternative for environmental valuation? Journal of Economic Surveys, 15(3), 435-462.

15. Hole, A. R., \& Kolstad, J. R. (2012). Mixed logit estimation of willingness to pay distributions: A comparison of models in preference and WTP space using data from a health-related choice experiment. Empirical Economics, 42(2), 445-469.

16. Kassie, G. T., Abdulai, A., Greene, W. H., Shiferaw, B., Abate, T., Tarekegne, A., \& Sutcliffe, C. (2017). Modeling preference and willingness to pay for drought tolerance (DT) in maize in rural Zimbabwe. World Development, 94, 465-477.

17. Lancaster, K. J. (1966). A new approach to consumer theory. Journal of Political Economy, 74(2), 132-157.

18. Mayer, J. E., Pfeiffer, W. H., \& Beyer, P. (2008). Biofortified crops to alleviate micronutrient malnutrition. Current Opinion in Plant Biology, 11(2), 166-170.

19. McFadden, D. (1974). The measurement of urban travel demand. Journal of Public Economics, 3(4), 303-328.

20. Mitchell, R., \& Carson, T. (n.d.). R.(1989). Using Surveys to Value Public Goods: The Contingent Valuation Method. Washington DC: Resources for the Future.

21. Nwanze, K. F. (2006). Rice development in sub-Saharan Africa. Journal of the Science of Food and Agriculture, 86(5), 675-677.

22. Owusu Coffie, R., Burton, M. P., Gibson, F. L., \& Hailu, A. (2016). Choice of rice production practices in Ghana: A comparison of willingness to pay and preference space estimates. Journal of Agricultural Economics, 67(3), 799-819.

23. Pambo, K. O. (2017). Analysis of consumer preference for Vitamin A-fortified sugar in Kenya. The European Journal of Development Research, 29(4), 745-768.

24. Quaye, W. (2010). Building the capacity of farmer based organization for sustainable rice farming in Northern Ghana. Journal of Agricultural Science, 2(1), 93-106.

25. Ritchie, H., \& Roser, M. (2017). Micronutrient deficiency. Our World in Data.

26. Rubey, L., Ward, R. W., \& Tschirley, D. (1997). Maize research priorities. The role of consumer preferences. Africa's Emerging Maize Revolution, 145-155.

27. Tschirley, D. L., \& Santos, A. P. (1995). Who eats yellow maize? Preliminary results of a survey of consumer maize preferences in Maputo, Mozambique.

28. Zimmermann, R., \& Qaim, M. (2004). Potential health benefits of Golden Rice: A Philippine case study. Food Policy, 29(2), 147-168. 